\section{Maternal Immune Activation in Pregnant Mice Produces Offspring with Altered Hippocampal Ripples}

\author{
Mengxuan Gao, ${ }^{a}$ Ken Orita, ${ }^{a}$ and Yuji Ikegaya ${ }^{*, a, b}$ \\ ${ }^{a}$ Graduate School of Pharmaceutical Sciences, The University \\ of Tokyo; Tokyo 113-0033, Japan: and ${ }^{b}$ Center for \\ Information and Neural Networks, National Institute of \\ Information and Communications Technology; Suita, Osaka \\ 565-0871, Japan. \\ Received January 12, 2019; accepted February 13, 2019
}

Psychiatric disorders, such as schizophrenia and autism spectrum disorder, are associated with sleep disturbances and deficits in memory consolidation; however, the relationship between these symptoms remains unclear. Here, we focused on hippocampal sharp-wave ripples (SWRs), a form of transient high-frequency oscillations that occur during sleep and behavioral immobility and contribute to memory consolidation. We activated the maternal immune system with polyinosinic-polycytidylic acid (poly(I:C)), one of the major pharmacological models of psychiatric disorders, to investigate whether SWR activity is altered in acute slices of the hippocampus from offspring born to poly(I: C)-treated mouse dams. Using robust continuous clustering in a low dimensional space defined by a uniform manifold approximation and projection, we found that mice with prenatal exposure to $\operatorname{poly}(\mathrm{I}: \mathrm{C})$ exhibited different feature distributions of SWR waveforms without affecting the overall frequencies of SWR events. Based on our results, maternal immune activation leads to altered SWR patterns in offspring.

Key words sharp wave; hippocampus; autism; infection; development

\section{INTRODUCTION}

Memory formation and consolidation rely on long-term modifications of the synaptic strength in the hippocampus and other brain regions. ${ }^{1,2)}$ Synaptic strength is potentiated by learning and experience during wakefulness, while it is downscaled to the baseline level during sleep to restore the capacity of the brain to acquire new memories. ${ }^{3,4)}$ During slow-wave sleep or awake immobility, hippocampal neurons fire spontaneously in brief periods and produce transient high-frequency oscillations $(150-300 \mathrm{~Hz})$, which are termed as sharp-wave ripple complexes (SWRs). ${ }^{5)}$ SWRs lead to a rescaling of synaptic strengths ${ }^{6,7)}$ and contribute to memory consolidation. ${ }^{8,9)}$

Patients with developmental neuropsychiatric disorders, such as schizophrenia and autism spectrum disorder (ASD), often exhibit impairments in sleep-dependent memory consolidation. ${ }^{10,11)}$ For example, persons with ASD show several abnormalities in sleep architecture, including decreased proportions of slow-wave sleep and reduced sleep spindles. ${ }^{12)}$ Since sleep spindles are associated with hippocampal SWRs in slow-wave sleep, ${ }^{13)}$ we hypothesized that hippocampal SWR activity is also altered in subjects with ASD and might account for the symptoms associated with memory consolidation deficits.

We utilized a mouse maternal immune activation (MIA) model using polyinosinic-polycytidylic acid (poly(I:C)), a synthetic analogue of double-stranded RNA, to test this hypothesis. MIA is one of the major environmental factors that cause psychiatric disorders in offspring. ${ }^{14)}$ Using acute brain slices prepared from the offspring of mouse dams that had been treated with saline or poly $(\mathrm{I}: \mathrm{C})$ during their gestational periods, we performed extracellular recordings to record local field potentials (LFPs) from the middle hippocampal CA1 region. We then classified SWRs using robust continuous clustering (RCC), an effective clustering algorithm based on the optimization of a continuous objective function, ${ }^{15}$ ) and evaluated and compared the features of the SWR waveforms between the two groups.

\section{MATERIALS AND METHODS}

Animal Ethics Animal experiments were performed with the approval of the Animal Experiment Ethics Committee at the University of Tokyo (approval number: P24-8) and according to the University of Tokyo guidelines for the care and use of laboratory animals. These experimental protocols were performed in accordance with the Fundamental Guidelines for Proper Conduct of Animal Experiment and Related Activities in Academic Research Institutions (Ministry of Education, Culture, Sports, Science and Technology, Notice No. 71 of 2006), the Standards for Breeding and Housing of and Pain Alleviation for Experimental Animals (Ministry of the Environment, Notice No. 88 of 2006) and the Guidelines on the Method of Animal Disposal (Prime Minister's Office, Notice No. 40 of 1995). All animals were housed on a 12-h dark-light cycle (lights on from 07:00 to 19:00) at $22 \pm 1^{\circ} \mathrm{C}$ with food and water provided ad libitum.

MIA Models Maternal viral infection was imitated by systemically administering poly(I:C) (Sigma, St. Louis, MO, U.S.A.) or saline (control) to pregnant C57BL/6J mice. Poly(I:C) was intraperitoneally injected at doses of 0.25 and $0.125 \mathrm{U} / \mathrm{g}$ on embryonic days 12.5 and 17.5 , respectively. ${ }^{16,17)}$ The pups produced by these poly(I:C)- or saline-treated mothers were used for experiments. Pups were removed from their dams at postnatal week 3 and were housed in groups of 2-4 animals per cage until postnatal week 4 .

Hippocampal Slice Preparations Acute slices were prepared from the hippocampus of 21- to 28-d-old mice of both sexes (SLC, Shizuoka, Japan). Mice were briefly anaesthetized with isoflurane, decapitated, and the brain was removed. Brains were placed in an ice-cold oxygenated solution containing (in mM): $27 \mathrm{NaHCO}_{3}, 1.5 \mathrm{NaH}_{2} \mathrm{PO}_{4}, 2.5 \mathrm{KCl}, 0.5$ ascorbic acid, $1 \mathrm{CaCl}_{2}, 7 \mathrm{MgSO}_{4}$, and 222.1 sucrose. Tissues were horizontally sliced at a thickness of $400 \mu \mathrm{m}$ and an angle of $12.7^{\circ}$ to the fronto-occipital axis using a Leica vibratome (VT1200S, Leica Microsystems, Nussloch, Germany). Slices were allowed to recover at $35^{\circ} \mathrm{C}$ for at least $90 \mathrm{~min}$ while submerged in a chamber filled with oxygenated artificial cerebrospinal fluid (aCSF) consisting of (in $\mathrm{mM}$ ): $127 \mathrm{NaCl}, 26$ $\mathrm{NaHCO}_{3}, 1.6 \mathrm{KCl}, 1.24 \mathrm{KH}_{2} \mathrm{PO}_{4}, 1.3 \mathrm{MgSO}_{4}, 2.4 \mathrm{CaCl}_{2}$, and 10 glucose. 

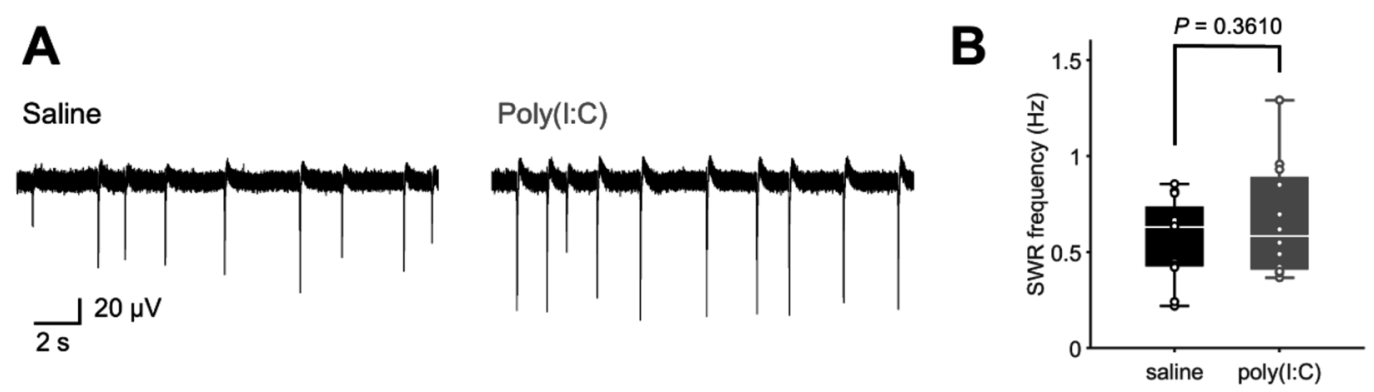

Fig. 1. Spontaneous SWRs in Acute Slices Prepared from Control or MIA Offspring

(A) Representative LFP traces recorded from the hippocampal CA1 pyramidal layer in brain slices prepared from offspring of mothers treated with saline or poly(I: C) during pregnancy. (B) Comparison of the frequencies of SWR events between the two groups. $n=12$ slices per group (6 slices from 4 male mice and 6 slices from 4 female mice for the saline group; 5 slices from 4 male mice and 7 slices from 5 female mice for the poly(I:C) group, respectively), $t_{22}=0.93$, Student's $t$-test after an $F$-test.

In Vitro Electrophysiological Recordings Slices were placed on $8 \times 8$ planar multi-electrode arrays (Alpha MED Scientific MED-P515A; electrode size, $50 \times 50 \mu \mathrm{m}$; inter-polar distance, $150 \mu \mathrm{m})$ and maintained in the interface condition at $35^{\circ} \mathrm{C}$. ACSF was bubbled with $95 \% \mathrm{O}_{2} / 5 \% \mathrm{CO}_{2}$ at room temperature and perfused at a rate of $1.0 \mathrm{~mL} / \mathrm{min}$. LFPs were simultaneously recorded from the 64 electrodes at $20 \mathrm{kHz}$ using the Alpha MED Scientific MED64 system. ${ }^{18)}$ For each slice, the trace recorded from the CA1 pyramidal layer with the highest amplitude was selected for subsequent analysis. We used custom-made MATLAB routines (The MathWorks, Natick, MA, U.S.A.) to analyze all data.

SWR Detection LFP traces were band-pass filtered between 2 and $30 \mathrm{~Hz}$, and SWRs were detected at a threshold below the means $-3 \times$ standard deviations (S.D.) of the baseline noise; notably, all SWRs were recorded as negative deflections in LFPs. During a post hoc visual inspection, events were manually rejected if they were erroneously detected as SWRs.

Feature Calculation For each SWR event, the peak point was determined as the minimum value in its raw trace, which represents the negative deflection (sink) peak. The peak height from the baseline was defined as the SWR amplitude. The times of the SWR onset and end were determined as the time points crossing a threshold of the mean $-1 \times$ S.D. in the raw trace. All data points below the threshold within -100 to $0 \mathrm{~ms}$ of the SWR peak were extracted. Of all data points whose last data points were greater than $0.05 \mathrm{~ms}$ above the threshold, the closest time to the SWR peak was defined as the onset time of the SWR event. Similarly, within 0 to $+100 \mathrm{~ms}$ of the SWR peak, data points below the threshold and whose next points were greater than $0.05 \mathrm{~ms}$ above the threshold were extracted, and the closest time to the peak was defined as the end time. The time interval between the onset and end points was defined as the SWR width. The SWR symmetry was defined using the following equation, rise time/(rise time + decay time), in which the rise time of the SWR is determined as the period required to deflect from 25 to $75 \%$ of the peak value in its raw trace, whereas the decay time is determined as the period required to deflect from 75 to $25 \%$. The raw trace within -13 to $+3 \mathrm{~ms}$ relative to the SWR peak time of the $2-30 \mathrm{~Hz}$ filtered trace was filtered between 150 and $300 \mathrm{~Hz}$ to define the degree of rippling, and the S.D. during this period was defined as the ripple strength.

Cluster Analysis and Visualization Datasets consisting of 4 parameters, the amplitudes, durations, symmetries and
A
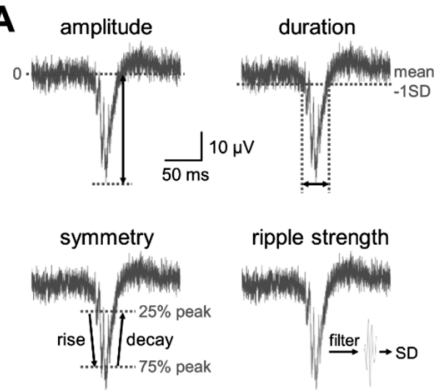

C

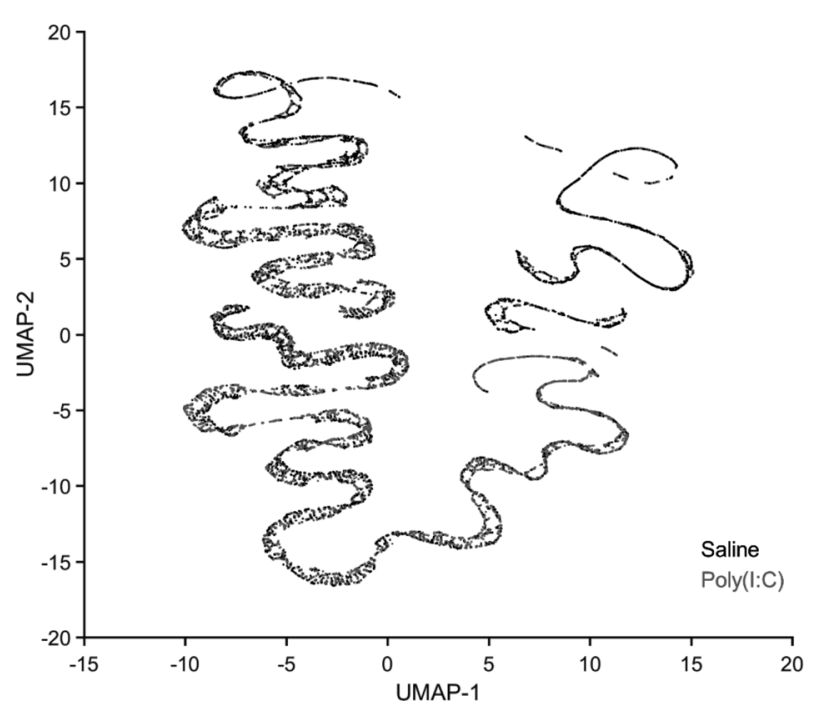

Fig. 2. Feature Extraction from SWR Waveforms

(A) Four parameters were measured to quantify the structures of an SWR event. Top left panel: the amplitude of an SWR is defined as the minimum value in its raw trace. Top right panel: the duration is defined as the period during which the raw trace fell below the mean $-1 \times$ S.D. Bottom left panel: symmetry is defined using the equation: rise time/(rise time + decay time); notably, the rise time of an SWR is defined as the period required to deflect from 25 to $75 \%$ of the peak value in its raw trace, whereas the decay time is defined as the period required to deflect from 75 to $25 \%$. Bottom right panel: the waveform around the peak of an SWR was filtered at $150-300 \mathrm{~Hz}$, and the S.D. value during this period is defined as the ripple strength. (B) A representative similarity matrix of SWRs observed in a hippocampal slice. The gray scale indicates the cosine similarity of four parameters between the pairs of SWRs $(1=$ identical and $0=$ no similarity). Seven hundred sixty-one SWRs occurred in this slice during the observation, and they were numbered in the temporal order of observation on the axes of the matrix. (C) Dimension reduction of the similarity matrix. All 17713 SWRs recorded in 24 slices were pooled, analyzed using UMAP, and plotted in space based on the first and second UMAP dimensions. Each dot indicates an SWR.

ripple strengths of SWRs were analyzed. The cosine similarity between 2 non-zero vectors, $\mathbf{A}$ and $\mathbf{B}$, was defined as the follows: 


$$
\text { similarity }=\cos \theta=\frac{\mathbf{A} \cdot \mathbf{B}}{\|\mathbf{A}\|\|\mathbf{B}\|}
$$

which ranges between -1 and 1; a higher value represents higher similarity. For a visualization of the data structure, we used a uniform manifold approximation and projection for dimension reduction (UMAP), ${ }^{19,20)}$ a nonlinear dimensionality reduction algorithm based on a combination of Riemannian geometry and algebraic topology for the metric realization of fuzzy simplistic sets. UMAP captures both local and global structures of the datasets. ${ }^{19)}$ We used unsupervised UMAP to transform the 4-dimensional features of SWRs into the 2-dimensional space (Python implemented with the default parameters $n$ neighbors $=15$, min dist $=0.1$, and metric $=$ 'euclidean'). For SWR classification, we used robust continuous clustering (RCC), ${ }^{15)}$ a clustering algorithm. RCC has a clear continuous objective and no prior knowledge of cluster numbers. We applied RCC to the 2-dimensional features obtained from UMAP (Python implemented with a clustering_threshold $=200, k=60$, and measure $=$ 'euclidean').

\section{RESULTS}

In the present study, we treated pregnant mice with

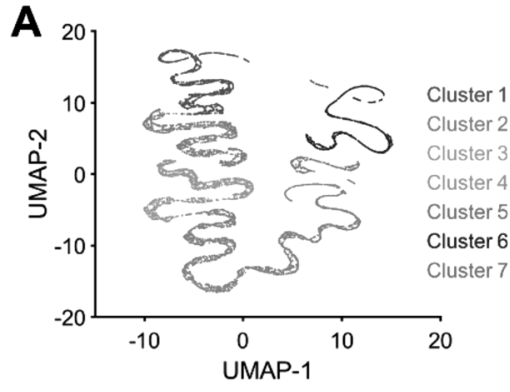

B Cluster 1

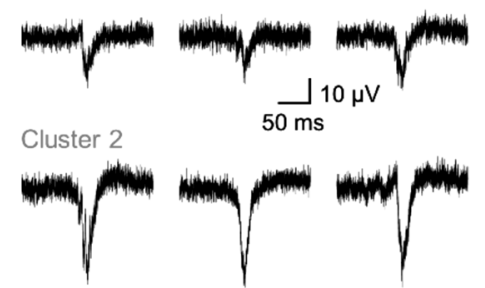

Cluster 3
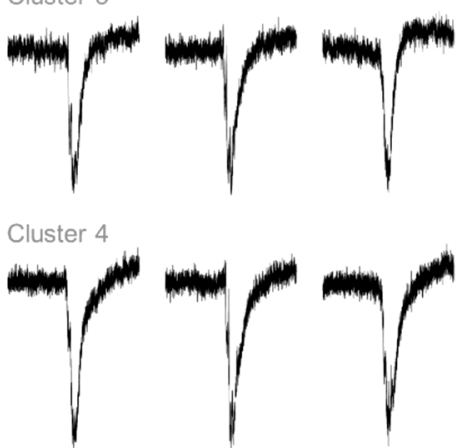

Cluster 5
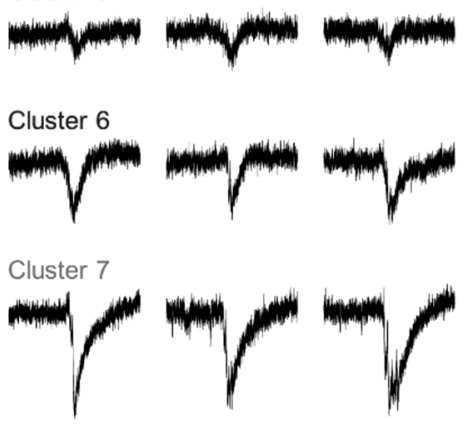

C
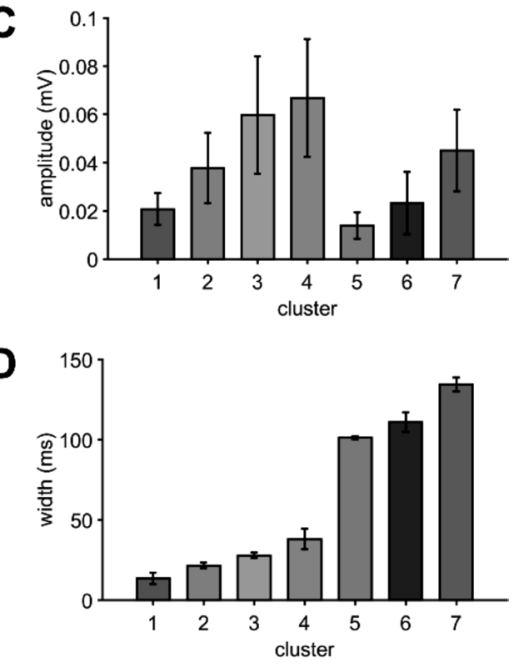

E

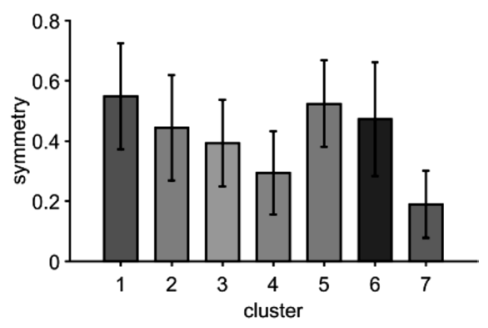

$\mathbf{F}$

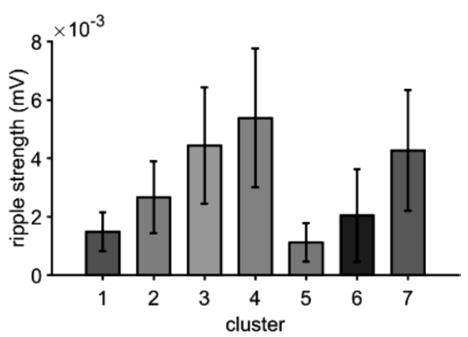

G

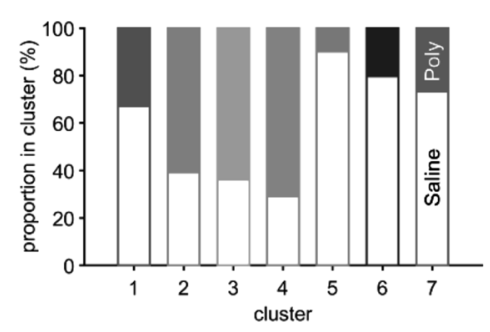

Fig. 3. Altered SWR Patterns in MIA Offspring

(A) RCC divided SWRs into 7 clusters, which are differently colored in the UMAP space. $n=2579,2902,3092,6305,763$, 1971, and 101 events, respectively, of each cluster. (B) Representative SWRs for each cluster. (C-F) Mean amplitudes (C), widths (D), symmetries (E) and ripple strengths (F) of SWRs in each cluster. The error bars represent standard deviations. (G) Stacked bar graph showing the proportions of SWRs recorded from saline-treated (open) or poly(I:C)-treated (filled) groups in each cluster. The proportions of SWRs recorded from the saline group were significantly different among clusters. $p<10^{-320}, \chi^{2}{ }_{(6)}=2894.3, \chi^{2}$ test. 
poly(I:C) to simulate virus infection events. In this MIA model, the mice are known to bear offspring with neurodevelopmental abnormalities associated with ASD and schizophrenia. ${ }^{21)}$ We used the offspring born from saline-treated (control) or poly(I:C)-treated dams for in vitro electrophysiological experiments at their young adult ages.

We prepared oblique brain slices, which spontaneously generate SWRs, possibly due to a more intact preservation of intrinsic neuronal circuits, to examine whether SWRs were altered in the MIA model. ${ }^{22}$ Slices prepared from offspring from both saline- and poly(I:C)-treated dams reliably exhibited SWR events in the hippocampal CA1 area (Fig. 1A) during a 20 -min recording period. The SWR event frequencies were $0.57 \pm 0.06 \mathrm{~Hz}$ for the saline group and $0.66 \pm 0.08 \mathrm{~Hz}$ for the poly(I:C) group (means \pm standard error of the mean (S.E.M.) of 12 slices from each group). The difference between two groups was not statistically significant (Fig. 1B, $p=0.36$, $t_{22}=0.93$, Student's $t$-test).

We evaluated the electrophysiological behaviors of SWRs using RCC, a clustering procedure. We extracted four features for each SWR: its amplitude, width, symmetry, and ripple strength (Fig. 2A). We collected 17713 SWRs from 24 slices (12 slices each from the saline and poly(I:C) groups) and created a $17713 \times 17713$ matrix for similarity between all possible pairs. In the matrix, each SWR was represented as a vector composed by the four features as described above, and the cosine similarity between each pair of SWRs was calculated and indicated in a gray scale. Part of the matrix (data from one representative slice) is shown in Fig. 2B. For illustration purposes, we applied UMAP to the matrix to reduce the four features of SWRs into the two-dimensional vector space (Fig. 2C).

Using RCC, we identified 7 clusters of SWRs (Fig. 3A). Cluster 4 was the largest, with a total of 6,305 SWR events, while clusters 1, 2, 3, and 6 contained 2579, 2902, 3092, and 1971 events, respectively. Clusters 5 and 7 were smaller clusters, containing 763 and 101 events, respectively. For each cluster, three representative raw traces of SWRs are displayed in Fig. 3B, and the means \pm S.D.s of the four features are shown in Figs. 3C-F. Differences in parameters were observed among these clusters, validating RCC-based classifications.

We then compared the proportions of SWRs for each cluster between the saline and poly(I:C) groups (Fig. 3G). The proportions varied significantly among clusters $\left(p<10^{-320}\right.$, $\chi_{(6)}^{2}=2894.3, \chi^{2}$ test); the saline group contributed more than $65 \%$ to clusters $1,5,6$, and 7 , whereas the poly(I:C) group was dominant in the other clusters. This separation indicates that SWRs in the poly(I:C) group tended to exhibit larger amplitudes, narrower widths, and stronger ripples than SWRs in the saline group.

\section{DISCUSSION}

Offspring born from poly(I:C)-treated dams produced SWRs with altered internal structures. More specifically, SWRs in the poly(I:C) group tended to distribute in clusters with greater amplitudes, narrower widths, and a greater ripple strength. Based on these results, neuronal activity is more synchronized during SWRs in the poly(I:C) MIA model than in the control group.

SWR activity increases after learning, possibly due to an increase in cellular excitability. ${ }^{23)}$ On the other hand, according to other studies, CA1 $\gamma$-aminobutyric acid (GABA)ergic interneurons and inhibitory postsynaptic currents are reduced in the poly(I:C) MIA models, suggesting an excitatory-toinhibitory imbalance in the neuronal activity of the hippocampus. ${ }^{17,24)}$ Therefore, highly synchronized SWRs in the poly(I:C) MIA models may reflect a hyperexcitatory state of the hippocampus, suggesting that synaptic weights are potentiated in poly(I:C) groups. SWRs trigger synaptic depression during slow-wave sleep, ${ }^{7)}$ which serves to reset unnecessarily potentiated synapses and thereby avoid memory saturation. Because we did not observe a difference in the event frequencies of SWRs between control and poly(I:C) groups, SWRs appear unlikely to induce synaptic depression in the poly(I:C) group. The lack of synaptic depression has two possible explanations. First, in the poly(I:C) group, synapses were less sensitive to SWRs as synaptic depression-inducing stimuli. This hypothesis is consistent with the fact that GABAergic function is affected in the poly(I:C) MIA model ${ }^{17,24)}$; notably, GABAergic inhibition is required to modulate synaptic plasticity. ${ }^{25)}$ Second, in the poly(I:C) group, SWRs lost the ability to induce synaptic depression. A theoretical model predicts that mild synaptic activation induces synaptic depression, whereas strong synaptic activation induces synaptic potentiation. ${ }^{26)}$ Slices in the poly(I:C) group produced highly synchronized SWRs, which might be too strong to induce synaptic depression. Further investigations are required to clarify these points.

In summary, offspring from poly(I:C)-treated mothers generated SWRs with large and sharp waveforms. These changes suggest a hyperexcited state of the hippocampus in the MIA models, which may affect synaptic modification and cause functional deficits in memory consolidation.

Acknowledgments This study was supported by JST ERATO (JPMJER1801), JSPS Grants-in-Aid for Scientific Research (18H05525), and the Human Frontier Science Program (RGP0019/2016). This work was conducted as part of a program at the International Research Center for Neurointelligence (WPI-IRCN) of The University of Tokyo Institutes for Advanced Study at The University of Tokyo.

Conflict of Interest The authors declare no conflict of interest.

\section{REFERENCES}

1) Lubenov EV, Siapas AG. Decoupling through synchrony in neuronal circuits with propagation delays. Neuron, 58, 118-131 (2008).

2) Whitlock JR, Heynen AJ, Shuler MG, Bear MF. Learning induces long-term potentiation in the hippocampus. Science, 313, 1093-1097 (2006).

3) Tononi G, Cirelli C. Sleep function and synaptic homeostasis. Sleep Med. Rev., 10, 49-62 (2006).

4) Tononi G, Cirelli C. Sleep and the price of plasticity: from synaptic and cellular homeostasis to memory consolidation and integration. Neuron, 81, 12-34 (2014).

5) Buzsáki G. Hippocampal sharp waves: their origin and significance. Brain Res., 398, 242-252 (1986).

6) Bukalo O, Campanac E, Hoffman DA, Fields RD. Synaptic plasticity by antidromic firing during hippocampal network oscillations. Proc. Natl. Acad. Sci. U.S.A., 110, 5175-5180 (2013). 
7) Norimoto H, Makino K, Gao M, Shikano Y, Okamoto K, Ishikawa T, Sasaki T, Hioki H, Fujisawa S, Ikegaya Y. Hippocampal ripples down-regulate synapses. Science, 359, 1524-1527 (2018).

8) Girardeau G, Benchenane K, Wiener SI, Buzsaki G, Zugaro MB. Selective suppression of hippocampal ripples impairs spatial memory. Nat. Neurosci., 12, 1222-1223 (2009).

9) Sadowski JH, Jones MW, Mellor JR. Sharp-wave ripples orchestrate the induction of synaptic plasticity during reactivation of place cell firing patterns in the hippocampus. Cell Reports, 14, 1916-1929 (2016).

10) Maski K, Holbrook H, Manoach D, Hanson E, Kapur K, Stickgold R. Sleep dependent memory consolidation in children with autism spectrum disorder. Sleep, 38, 1955-1963 (2015).

11) Wamsley EJ, Tucker MA, Shinn AK, Ono KE, McKinley SK, Ely AV, Goff DC, Stickgold R, Manoach DS. Reduced sleep spindles and spindle coherence in schizophrenia: mechanisms of impaired memory consolidation? Biol. Psychiatry, 71, 154-161 (2012).

12) Limoges E, Mottron L, Bolduc C, Berthiaume C, Godbout R. Atypical sleep architecture and the autism phenotype. Brain, 128, 1049-1061 (2005).

13) Sirota A, Csicsvari J, Buhl D, Buzsaki G. Communication between neocortex and hippocampus during sleep in rodents. Proc. Natl. Acad. Sci. U.S.A., 100, 2065-2069 (2003).

14) Atladóttir HO, Thorsen P, Ostergaard L, Schendel DE, Lemcke S, Abdallah M, Parner ET. Maternal infection requiring hospitalization during pregnancy and autism spectrum disorders. J. Autism Dev. Disord., 40, 1423-1430 (2010).

15) Shah SA, Koltun V. Robust continuous clustering. Proc. Natl. Acad. Sci. U.S.A., 114, 9814-9819 (2017).

16) Naviaux RK, Zolkipli Z, Wang L, Nakayama T, Naviaux JC, Le TP, Schuchbauer MA, Rogac M, Tang Q, Dugan LL, Powell SB. Antipurinergic therapy corrects the autism-like features in the poly(I:C) mouse model. PLOS ONE, 8, e57380 (2013).
17) Okamoto $\mathrm{K}$, Hitora-Imamura N, Hioki H, Ikegaya Y. GABAergic malfunction in the anterior cingulate cortex underlying maternal immune activation-induced social deficits. J. Neuroimmunol., 321, 92-96 (2018).

18) Gao M, Igata H, Takeuchi A, Sato K, Ikegaya Y. Machine learningbased prediction of adverse drug effects: an example of seizureinducing compounds. J. Pharmacol. Sci., 133, 70-78 (2017).

19) Becht E, McInnes L, Healy J, Dutertre CA, Kwok IWH, Ng LG, Ginhoux F, Newell EW. Dimensionality reduction for visualizing single-cell data using UMAP. Nat. Biotechnol., 37, 38-44 (2018).

20) McInnes L, Healy J, Melville J: UMAP: uniform manifold approximation and projection for dimension reduction. arXiv, 1802, 03426 (2018).

21) Patterson PH. Modeling autistic features in animals. Pediatr. Res., 69, 34R-40R (2011).

22) Mizunuma M, Norimoto $H$, Tao K, Egawa $T$, Hanaoka $K$, Sakaguchi T, Hioki H, Kaneko T, Yamaguchi S, Nagano T, Matsuki N, Ikegaya Y. Unbalanced excitability underlies offline reactivation of behaviorally activated neurons. Nat. Neurosci., 17, 503-505 (2014).

23) Eschenko O, Ramadan W, Molle M, Born J, Sara SJ. Sustained increase in hippocampal sharp-wave ripple activity during slow-wave sleep after learning. Learn. Mem., 15, 222-228 (2008).

24) Ducharme G, Lowe GC, Goutagny R, Williams S. Early alterations in hippocampal circuitry and theta rhythm generation in a mouse model of prenatal infection: implications for schizophrenia. PLOS ONE, 7, e29754 (2012).

25) Paulsen O, Moser EI. A model of hippocampal memory encoding and retrieval: GABAergic control of synaptic plasticity. Trends $\mathrm{Neu}-$ rosci., 21, 273-278 (1998).

26) Bienenstock EL, Cooper LN, Munro PW. Theory for the development of neuron selectivity: orientation specificity and binocular interaction in visual cortex. J. Neurosci., 2, 32-48 (1982). 